\title{
Evolución y magnitud de la pobreza en México
}

\author{
Julio Boltvinik*
}

\begin{abstract}
El presente trabajo destaca las caracteristicas del método de medición integrada de la pobreza (ммIP), en esta que constituye una de sus primeras aplicaciones, proporcionando una visión de la magnitud y características de la pobreza en México. Utilizando el método de linea de pobreza $\left.{ }_{(L P}\right)$, en su variante de canasta normativa de satisfactores esenciales (CNSE), se muestra que mientras la incidencia de la pobreza disminuyó durante el periodo 1963-1981, la tendencia se revirtió en 1982, y desde entonces no ha dejado de crecer. Tanto este análisis como el que se aplica para 1989 con base en el MMIP, muestran que la gravedad de la pobreza es mucho mayor que la que suele considerarse: más de dos terceras partes de los mexicanos son pobres y cerca de la mitad (45\%) son pobres extremos. Aunque hay más personas pobres en el medio urbano que en el rural, al tomar en cuenta también la intensidad de sus carencias, se concluye que hay más pobreza en el campo. Desde el punto de vista metodológico se constata la capacidad analítica del ммIP y se puede aseverar que, conforme a este método, el nivel de incidencia de la pobreza se ubica entre el determinado por los métodos parciales de necesidades básicas insatisfechas (NBr) y de LP.
\end{abstract}

\section{Introducción**}

Este artículo persigue un doble propósito. Por una parte, presentar el primer cálculo empírico que se haya hecho aplicando un procedimiento nuevo para la estimación de la pobreza, el método de medición integrada de la pobreza (ммір) (Boltvinik, 1992), que tiene un propósito metodológico: destacar sus características y contrastarlo con los procedimientos usuales. Por otra parte, intenta proporcionar una imagen lo más completa posible sobre la evolución y las principales características de la pobreza en el país.

En la primera parte se presenta una recopilación sintética de los métodos de medición de la pobreza utilizados: el de línea de pobreza (LP) en su variante canasta normativa de satisfactores esenciales (CNSE), con base en el cual están construidos los cálculos de evolución de la pobreza, y el mir que ha sido utilizado para presentar los cálculos de la pobreza correspondientes a 1989.

* Profesor-investigador del Centro de Estudios Sociológicos, El Colegio de México.

** Los resultados aquí presentados se derivan de dos trabajos míos anteriores. Por un lado, recojo de J. Boltvinik (1994) el breve análisis de la evolución de la pobreza que presento en la segunda parte y, en la tercera parte, retomo del capitulo 5 de J. Boltvinik (en prensa) el cálculo de la magnitud de la pobreza en 1989 y el análisis de sus dimensiones fundamentales. 
En la segunda parte se muestra la evolución de la pobreza en México desde 1963 hasta 1992 tomando como base las mejores estimaciones disponibles.

En la tercera parte se exponen los resultados de la aplicación del MMIP. Aunque se elaboraron los cálculos desagregados para cuatro tipos de localidad y para cinco regiones de carácter no contiguo (o estratos), estos últimos no se presentan. Para cada uno de dichos tipos de localidades y regiones, así como para el total nacional, se realizó el cálculo de la población que pertenece a cada uno de los estratos en los que se clasificó a los hogares nacionales y a sus ocupantes, partiendo de cuatro opciones metodológicas: los métodos parciales de línea de pobreza-tiempo (LPT) y de necesidades básicas insatisfechas (NBI), y las dos maneras dominantes de integrarlos: por medio del MMIP y de lo que he llamado el MMip en su variante burda o matricial.

El MMIP unifica todas las dimensiones del bienestar en una medición única e integrada de la pobreza; por tanto, constituye la más adecuada de sus medidas. Las otras mediciones parciales (incluyendo la que se presenta en la segunda parte: la de LP-CNSE), así como el MMIP matricial, permiten desagregar el MMIP para entenderlo mejor; pero las cifras que establecen la magnitud de la pobreza en México son exclusivamente las del MMIP.

En la cuarta parte se presentan algunas conclusiones, tan to de carácter empírico como metodológico.

\section{Los métodos utilizados}

El método de línea de pobreza basado en la canasta normativa de satisfactores esenciales (CNSE)

Como parte de los trabajos de investigación de Coplamar, durante los años de 1982 y 1983, desarrollé la variante de la CNSE. El primer paso de esta metodología consiste en determinar los requerimientos de bienes y servicios de los individuos que conforman un hogar durante un periodo determinado, por ejemplo un año. En el caso de los bienes durables es necesario distinguir entre la cantidad requerida y el uso familiar anual, siendo el primero mayor que el segundo. Por ejemplo, la familia requiere una estufa, pero sólo usa -desgasta o deprecia- 0.10 estufas anualmente. En los no durables, por ejemplo los alimentos, las cifras son iguales. La verdadera canasta está definida 
por el vector de cantidades del uso anual. Es éste el que debe multiplicarse por los precios de los bienes para obtener el costo anual de cada rubro. La suma de los costos anuales de todos los rubros constituye el costo anual de la cNsE. Éste constituye la línea de pobreza, que se compara con los ingresos o el consumo del hogar para definir si el hogar es o no pobre.

En el cálculo de los requerimientos normativos surgen dos problemas fundamentales. En primer lugar, la sustentación de las normas de las que dichos requerimientos se derivan; éste es el problema más complejo en los estudios de pobreza y sobre el que menos acuerdo existe. Una de las vertientes de la discusión, entre las concepciones absoluta y relativa de la pobreza, puede verse como la polémica entre dos:juicios respecto a los umbrales mínimos debajo de los cuales se presentan las situaciones de pobreza: la de sobrevivencia física y la de normas socialmente determinadas. ${ }^{I}$ En la construcción de la cNSE se partió de un doble criterio: por una parte, se consideró la realidad del país reflejada en los bienes y servicios de consumo frecuente; por otra, la legislación que expresa tanto realidades como objetivos por alcanzar. La instrumentación del primer concepto se logró por medio del análisis de las prácticas de consumo del decil 7 de la población nacional según la Encuesta Nacional de Ingresos y Gastos de los Hogares (ENIGH) de 1977. El segundo criterio consideró los derechos que la legislación otorga, tanto los sociales - para todos los habitantes-como los de clase. Con estas bases se llegó a una definición -la CNSE- que expresa un concepto de pobreza relativa.

En segundo lugar, la lista de requerimientos totales debe clasificarse en dos grupos. Por una parte, aquellos que habrán de ser satisfechos por la vía del consumo privado, es decir, cuyo costo deberá ser sufragado por las familias; y por otra, el que habrá de ser financiado por el gasto público (gubernamental o de instituciones de seguridad social). Sólo los rubros del primer tipo deben conformar la línea de pobreza, puesto que ésta se compara con los ingresos o con los gastos de consumo privado de los hogares. Aquí caben dos procedimientos. El más simple consiste en definir para todos los hogares, de manera idéntica, aquellos rubros comprendidos en cada categoría. Otro, más arduo, consiste en formar esta clasificación para cada hogar según sus condiciones particulares de acceso a las transferencias públicas. En la cNSE

\footnotetext{
1 Para una síntesis de la discusión, véase J. Boltvinik, (1990: 26-30).
} 
de Coplamar se adoptó el primer camino: los satisfactores que habrían de cubrirse con cargo al gasto gubernamental o de la seguridad social, fueron los servicios de educación primaria y secundaria, tanto para menores como para adultos, la prestación de los servicios de salud, y la instalación y mantenimiento de la infraestructura de agua y drenaje. El acceso a estos servicios sería entonces por la vía de las transferencias públicas. El resto de los rubros debería ser financiado por los hogares, lo cual supone su acceso por las vías mercantil o de autoproducción. ${ }^{2}$ Esta división única y para todos los hogares facilitó los cálculos, pero subestimó el nivel de la línea de pobreza. Esto es así ya que, por ejemplo, la población que no tiene acceso a los servicios de salud gratuitos se ve obligada a sufragar los gastos en la materia, pero éstos no se contemplan en la línea de pobreza.

La LP resultante se compara entonces con los ingresos de los hogares. Aquí nuevamente hicimos una simplificación en Coplamar. El cálculo de la línea de pobreza se refirió a la familia promedio de cada decil, según el tamaño y composición media de sus hogares. Lo más exacto es hacer esto para cada hogar, lo que se puede lograr calculando la CNSE por persona o por adulto equivalente, de tal manera que la LP aplicable a cada uno resulte de multiplicar este costo unitario por el número de unidades en cada hogar.

\section{El método de medición integrada de la pobreza (MмIP)}

Rasgos generales del método y del procedimiento específico utilizado

El MMIP combina dos metodologías previamente existentes. Por una parte, la de LP en su variante de CNSE, y por la otra, la de necesidades básicas insatisfechas (NBI) en su versión mejorada (véanse Boltvinik, 1992; Gobierno de la República de Bolivia, 1993). Su fundamento es la siguiente concepción de las fuentes de bienestar de los hogares y la crítica de los métodos de LP y de NBI a partir de ésta:

Dadas sus necesidades, cuya variabilidad se suele subestimar, la satisfacción de las necesidades básicas de una persona o de un hogar, depende

2 Para el análisis de las formas de acceso a los satisfactores, véanse J. Boltvinik 1984,1986 y $1986 a$. 
de las siguientes seis fuentes de bienestar: $a$ ) el ingreso corriente; $b$ ) los derechos de acceso a servicios o bienes gubernamentales de carácter gratuito (o subsidiados); c) la propiedad, o derechos de uso, de activos que proporcionan servicio de consumo básico (patrimonio básico acumulado); $d$ ) los niveles educativos, las habilidades y destrezas, entendidos no como medios de obtención de ingresos, sino como expresiones de la capacidad de entender y hacer; $\theta$ ) el tiempo disponible para la educación, la recreación, el descanso, y para las labores domésticas, y ff) los activos no básicos o la capacidad de endeudamiento del hogar.

Entre algunas de estas fuentes de bienestar existe posibilidad de sustitución. Con un mayor ingreso se pueden sustituir algunos derechos de acceso, atendiendo necesidades como salud y educación privadamente, o sustituir la no propiedad de algunos activos de consumo (verbigracia, rentar una vivienda). Esta sustituibilidad no es perfecta, sin embargo. Con ingresos adicionales no se puede sustituir la falta de tiempo disponible para educación y recreación; si no están desarrolladas las redes básicas de agua y drenaje, no será posible (o será muy caro) acceder a estos servicios.

La limitación principal de los métodos de línea de pobreza y de necesidades básicas insatisfechas (tal como éste se ha venido aplicando en América Latina) consiste en que proceden, el primero, como si la satisfacción de necesidades básicas dependiera solamente del ingreso o del consumo privado corriente de los hogares; el segundo, en sus aplicaciones usuales (haciendo caso omiso del último indicador), elige indicadores de satisfacción de necesidades que básicamente dependen en América Latina de la propiedad de activos de consumo (vivienda) o de los derechos de acceso a servicios gubernamentales (agua, eliminación de excretas y educación primaria), por lo cual implícitamente deja de tomar en cuenta las demás fuentes de bienestar.

Es decir, el método de LP no toma en cuenta las fuentes $b$ ) a $f$ ) cuando se compara la línea de pobreza con el ingreso del hogar, o las fuentes $b$ ) a $e$ ) cuando se compara con el consumo. Por su parte, el método de NBI, tal como se ha venido aplicando en América Latina, deja de considerar el ingreso corriente y las fuentes $d$ ) a $f$ ). Es decir, ambos tienen una visión parcial de la pobreza, por lo cual tienden a subestimarla. En la medida en que las fuentes de bienestar consideradas por ambos métodos son distintas, de inmediato podemos concluir que más que procedimientos alternativos, $\mathrm{co}^{-}$ mo se les suele considerar, son complementarios (J. Boltvinik, 1992: 355).

El mMip se desarrolla para considerar de manera cabal estas fuentes de bienestar de las personas. Para lograr la plena complementariedad de los dos métodos en los cuales se apoya, se requiere precisar cuáles necesidades se detectarán por el método de NBI y cuáles por LP. En principio, deberían trabajarse por NBI todas las que dependan conceptualmente o de manera preponderante -y para la mayoría de los hogares- del gasto público (consumo e inversión), de la inversión acumulada del hogar, y del tiempo disponible de las personas del ho- 
gar. Quedarían para ser analizadas por I.P las necesidades que dependan fundamentalmente del consumo privado corriente.

En consecuencia, debería identificarse por NBI la satisfacción de las siguientes necesidades:

i) Los servicios de agua y drenaje.

ii) El nivel educativo de los adultos y la asistencia escolar de los menores.

iii) La electricidad.

iv) La vivienda.

v) El mobiliario y equipamiento del hogar.

vi) El tiempo libre para recreación.

Los casos de atención a la salud y a la reproducción biológica, y de seguridad (social), puesto que pueden satisfacerse mediante servicios gratuitos o privados, requieren un tratamiento mixto. Si las personas no tienen acceso a los servicios gratuitos, y su ingreso no les permite la atención médica privada y el acceso a seguros privados, las necesidades en cuestión se considerarán insatisfechas.

Quedarían como necesidades cuya satisfacción-insatisfacción se verificaría exclusivamente por L.P, las de:

vii) Alimentación.

viii) Vestido, calzado y cuidado personal.

ix) Higiene personal y del hogar.

$x)$ Transporte y comunicaciones básicas.

xi) Adicionalmente, casi todas las necesidades identificadas por NBI conllevan gastos corrientes por parte del hogar, que deben ser considerados para fijar el nivel de la línea de pobreza.

xii) Las necesidades de recreación, información y cultura imponen a las familias requisitos de tipo mixto. Por una parte es necesaria la disponibilidad de tiempo, pero por otra casi siempre resulta necesario incurrir en una serie de gastos (equipo para hacer deporte, boletos para espectáculos, gastos de transporte, etcétera). La solución ideal sería identificar directamente su (in) satisfacción. No es tampoco mala solución identificar la disponibilidad de tiempo libre por NBI e incorporar gastos monetarios requeridos a la línea de pobreza.

xiii) En los hogares donde todos o algún(os) miembros carecen de acceso a servicios gratuitos de salud y a cobertura de la seguridad social, el costo privado de atención de estas necesidades se incluirá en la L.P y/o el gasto realizado se descontará del ingreso antes de compararlo con la LP. 
El procedimiento general de NBI mejorado empieza construyendo un indicador de logro, lo que supone el otorgamiento de puntajes a variables como las antes citadas, así como la definición de la norma mínima en cada dimensión. Este indicador se estandariza al dividirlo entre el puntaje de la norma, de tal manera que la variable queda expresada en unidades de ésta. Con ello la variable pierde la unidad de medida original en la que estaba expresada y se convierte en un número puro. El siguiente paso es uniformar, al máximo posible, el rango de variación de los indicadores estandarizados, para lo cual se reescalan los valores superiores a la norma -cuando el máximo observable rebase el valor 2- para acotarlos entre más de 1 y 2 . Con ello se busca que todos los indicadores de logro queden en el rango de 0 a 2 , con la norma en el 1. El último paso es reconvertir este indicador a uno de carencia, restando su valor de 1 y dejando el rango de los indicadores de carencia entre -1 y +1 , con la norma en el 0 . Los valores positivos expresan carencias, el 0 equilibrio, y los valores negativos, bienestar. Lamentablemente no en todos los indicadores se pudo lograr el rango total de variación, por lo que algunos de ellos son indicadores de carencia que sólo varían del $0 \mathrm{al}+1$.

En la aplicación aquí presentada, seis dimensiones se consideraron por el método de NBI o método directo, y una por un procedimiento mixto (salud y seguridad social). Las seis dimensiones de NBI son:

i) Inadecuación de la calidad y cantidad de la vivienda, que se forma, a su vez, de 2 subdimensiones: inadecuación de la calidad de la construcción e inadecuación del espacio de la vivienda. La variable de logro de la primera subdimensión se construye como una media ponderada de los cocientes entre los puntajes otorgados a los materiales utilizados en muros, techos y pisos y los puntajes de las normas respectivas. La norma de muros se fijó en los construidos con tabique, ladrillo, block, piedra o cemento (puntaje 2). Los muros de madera y adobe recibieron puntaje 1; los de carrizo, bambú, o palma, barro o bajareque, lámina metálica o de asbesto, recibieron puntaje 0.5. El puntaje de los muros de lámina de cartón y de otros materiales fue 0 . La variable de logro de la segunda subdimensión expresa el inverso del hacinamiento y se mide por la relación entre cada uno de los tipos de espacios de la vivienda (dormitorios, cocina y cuartos multiuso) y el número de sus ocupantes. El índice se construye dividiendo el número de dormitorios equivalentes observados en la vivienda entre el número de dormitorios equivalentes normativos. Los dormitorios equivalentes se obtienen valuando la cocina como 0.5 dormitorios y los cuartos multiuso 
(definidos operacionalmente como los que no son cocina y no se usan para dormir) como 1.5 dormitorios. Las normas se definieron como un dormitorio para cada 2 personas y un multiuso para cada 4 en zonas urbanas; $y$ un dormitorio para cada 2.5 personas y un multiuso para cada 5 en zonas rurales. Se exige cocina de uso exclusivo para todos los hogares de dos o más personas. El índice sintético de inadecuación de la vivienda resulta de la multiplicación de los indicadores de las dos subdimensiones.

ii) Inadecuación de las condiciones sanitarias, que se integra por los indicadores de agua, drenaje y baño. La norma de agua se fijó en la opción "entubada dentro de la vivienda", recibiendo las demás opciones valores crecientemente menores a la norma, mientras más se alejan de ella. En drenaje se fijó la norma en la opción de "drenaje conectado al de la calle o a fosa séptica", quedando con valor 0 la variable de logro cuando la opción observada era "no dispone de drenaje o con desagüe al suelo, a un río o un lago". La norma de baño se fijó en la opción "baño exclusivo con conexión de agua". El indicador consolidado de adecuación sanitaria es la media ponderada, según costos, de los tres indicadores.

iii) Inadecuación de otros servicios, que se integra por los indicadores de electricidad y teléfono. El teléfono sólo se exigió para las ciudades de 500000 o más habitantes. Ambas son variables de logro dicotómicas que reciben puntaje 1 cuando están presentes y 0 cuando no lo están. En las metrópolis el indicador de otros servicios es la media ponderada, por costos, de los indicadores de teléfono y electricidad.

iv) Inadecuación del patrimonio básico, que constituye un indicador de una de las fuentes de bienestar, y no de una necesidad específica en particular (algo enteramente similar a lo que ocurre con el ingreso). En la norma se incluyen equipos domésticos asociados a las necesidades de alimentación, higiene y recreación, entre otras. El valor del indicador para un hogar se obtiene dividiendo el valor de los bienes durables que posee cada hogar entre el valor de los equipos incluidos en la norma. Los valores de cada aparato se fijaron en función del modelo y marca más pequeño y barato.

v) Rezago educativo, que se construye a partir de los indicadores de alfabetismo y grado de instrucción. El primero se usa como variable de control y el indicador básico se construye a partir del cociente entre grados aprobados y grados normativos según la edad de la persona. La norma para los adultos de 15 a 49 años es de secundaria completa o equivalente (nueve grados). 
vi) Exceso de tiempo de trabajo, como indicador inverso de tiempo disponible para educación, recreación y trabajo doméstico (también indicador de una de las fuentes de bienestar). Se construye a partir de la norma de 48 horas a la semana para todos los miembros del hogar que están en posibilidades de llevar a cabo trabajo extradoméstico y que se calcula restando de los adultos de 15 a 69 años los excluidos por jubilación, estudio y requerimientos de trabajo doméstico, entre otras razones.

El procedimiento mixto se utiliza en el siguiente indicador:

vii) Inadecuación de acceso a la atención a la salud y a la seguridad social. Éste se calcula otorgando el valor de la norma a los derechohabientes de la seguridad. A los no derechohabientes se les asignó el valor de la norma cuando su ingreso estaba entre dos y tres veces la línea de pobreza, monto que se calculó como necesario para cubrir el valor del seguro de gastos médicos mayores y para generar un ahorro suficiente para cubrir los riesgos de vejez, invalidez y similares.

La satisfacción de las demás necesidades se identifica por el método indirecto o de la línea de pobreza, comparando el ingreso por adulto equivalente de los hogares, con las líneas de pobreza y de pobreza extrema también expresadas por adulto equivalente.

Se obtienen así seis indicadores de carencia por NBI, uno mixto y uno de LP para cada hogar. Los indicadores sintéticos de cada una de las cinco primeras dimensiones de NBI y el indicador mixto se combinan mediante una media aritmética ponderada para obtener el indicador integrado de NBI en cada hogar, que indica el grado de insatisfacción del conjunto de las necesidades verificadas directamente, $o$ intensidad de la pobreza por NBI: $\mathrm{I}(\mathrm{NBI})_{j}$. Por otra parte, el indicador de exceso de trabajo y el de ingresos se combinan en un indicador compuesto de tiempo-ingresos, que resulta de dividir el ingreso entre un índice de exceso de tiempo de trabajo, antes de compararlo con la LP, para obtener la intensidad de la pobreza por ingresos-tiempo: I(LPT) ${ }_{\mathrm{j}}$. Para integrar las cinco dimensiones de NBI y la mixta entre sí, así como su indicador sintético con el de la dimensión ingresos-tiempo, se utiliza un sistema de ponderadores de costos que se deriva de la estructura de costos que provee la cNSE.

Al integrar así las dimensiones de I.P-tiempo, I(LPT), y el de NBI, I(NBI), se obtiene el indicador integrado de I(MMIP) para cada hogar, que indica si éste es pobre o no y la intensidad de su pobreza.

Una vez identificada la población pobre y la no pobre, tanto por cada uno de los métodos parciales como por el integrado, se procede a: 
a) clasificar la población pobre en tres estratos, según la intensidad de su pobreza, y a la población no pobre en otros tres estratos según sus condiciones de riqueza.

b) Para cada estrato y para el conjunto de la población pobre, se calculan los principales índices de pobreza: la incidencia $(\mathrm{H})$, la intensidad (I), y el grado (HI).

c) Finalmente, se presenta el perfil de las carencias de cada estrato.

\section{Desagregación geográfica del análisis}

La ENIGH de 1989 se apoya en una muestra nacional. Una muestra complementaria proporciona resultados adecuados para el Área Metropolitana de la Ciudad de México (АмСм). Esto divide el total "urbano"3 en dos partes: por una, el Амсм y por la otra, el resto urbano. Adicionalmente, el resto urbano se divide en ciudades de 500000 y más habitantes, y menores de esta cantidad. Por otra parte, aunque obtuve cálculos para un grupo de regiones no contiguas o estratos geográficos, homogeneizados según la incidencia en ellos de la pobreza extrema, por razones de espacio no se presentan aquí.

\section{Evolución de la pobreza en México}

\section{Evolución de la pobreza por ingresos según el método de LP-CNSE}

Aplicando la cNSE, cuya construcción expliqué anteriormente en términos generales, Enrique Hernández Laos (1992) ha calculado la incidencia de la pobreza y de la pobreza extrema en México en 1963, 1968,1977 y $1984 .{ }^{4}$ Asimismo, con ciertos supuestos, ha hecho una estimación de la pobreza en 1981 y en 1988 . Esto permite tener una

3 La ENIGH de 1989 sustituye los conceptos de urbano y rural por los de alta y baja densidad. La zona de alta densidad incluye los municipios que cumplen una o más de las siguientes características: tener por lo menos una localidad de 15000 o más habitantes; una población total mayor o igual a 100000 habitantes; contener a la capital de la entidad federativa; o formar parte de alguna de las doce zonas metropolitanas del país.

4 En el texto que sigue se omite el análisis de la pobreza extrema, porque la manera en que la mide Hernández Laos, que apliqué en Coplamar en 1982, me parece ahora inadecuada. La población comprendida dentro de la pobreza extrema fue deffnida como aquella que tiene ingresos inferiores al costo de una canasta llamada submí- 
apreciación de largo plazo de la evolución de la pobreza en el país. En esta sección reseño los resultados de su análisis y, a la luz de información reciente sobre la distribución del ingreso, discuto los posibles niveles de la pobreza en México en 1989 y 1992. En el cuadro 1 resumo los resultados obtenidos por Hernández Laos y añado mis propias estimaciones.

\section{CUADRO 1}

Evolución de la pobreza en México 1963-1992, según el método de línea de pobreza. Variante: canasta normativa de satisfactores esenciales (porcentaje de la población nacional)

\begin{tabular}{llllllllr}
\hline & 1963 & 1968 & 1977 & $1981^{\mathrm{e}}$ & 1984 & $1988^{\mathrm{e}}$ & $1989^{\mathrm{e}}$ & $1992^{\mathrm{e}}$ \\
\hline Total de pobres & 77.5 & 72.6 & 58.0 & 48.5 & 58.5 & 59.0 & 64.0 & 66.0 \\
Tasa media anual & & $(1.3)$ & $(2.5)$ & $(4.6)$ & 6.5 & & 1.8 & 1.0 \\
\hline
\end{tabular}

${ }^{\mathrm{e}}$ Cifras estimadas. En 1981 y 1988 por Hernández Laos. En 1989 y 1992 por Julio Boltvinik.

Fuentes: Para 1963, 1968, 1977, 1981 y 1988, véase Hernández Laos, 1992. Para 1989 y 1992 , se trata de estimaciones propias.

Las tendencias mostradas en este cuadro son las de una rápida reducción de la proporción de población en situación de pobreza en el periodo 1963-1968 (a una tasa media anual de -1.3\%), una muy rápida baja entre 1968 y 1977 (-2.46\% anual) y una aceleradísima disminu-

nima, que corresponde a los rubros de alimentación, vivienda, salud e higiene, y educación de la CNSE. Esta manera de cortar los satisfactores de una canasta es inadecuada, ya que se eliminan necesidades completas, como transporte, vestido y calzado, en las cuales incluso los más pobres tienen que gastar algo. La manera correcta de acercarse a una conceptuación de la pobreza extrema y a una medición coherente de ella, consiste en desarrollar y hacer operable la distinción entre pobreza absoluta y relativa, incluyendo en la primera solamente lo indispensable para la satisfacción de todas las necesidades en condiciones de dignidad, mientras que en la segunda se incluyen además los elementos de participación en el estilo de vida socialmente dominante y de reducción del trabajo doméstico extremo. He emprendido esta vía, de manera experimental, en mis trabajos de medición de la pobreza citados en la nota 1. La canasta de satifactores que permite calcular la línea de pobreza extrema, a la que he llamado canasta normativa de satisfactores de subsistencia (cNss), tiene un carácter horizontal, esto es, comprende todas las necesidades, pero en cada una de ellas selecciona exclusivamente los rubros estrictamente indispensables para satisfacerlas en condiciones de dignidad. Por ejemplo, la cNss no incluye previsión alguna para el consumo de alimentos fuera del hogar, ni incluye el refrigerador doméstico. 
ción entre 1977 y 1981 (aunque en este caso se trata de una estimación, ya que no se cuenta con una encuesta de ingresos y gastos para 1981): $4.6 \%$ anual. Así, se habría logrado reducir en 18 años la pobreza de más de las tres cuartas partes de la población a menos de la mitad.

Sin embargo, después de 1981 habría ocurrido un brusco cambio de tendencia por el cual la pobreza no sólo habría dejado de disminuir sino que habría empezado a aumentar aceleradamente, alcanzando tres años después $58.5 \%$, lo que significaría una tasa media anual de crecimiento de $6.5 \%$. El porcentaje de pobreza en 1984 es ligeramente superior al de 1977.

Para estimar la pobreza en 1981 y en 1988, el autor aplicó al primer año la estructura por deciles de la distribución del ingreso de 1977 y la de 1984 para el segundo. Es decir, ambas son estimaciones de lo que habría sido la pobreza en el país si hubiera prevalecido la distribución supuesta. En ese caso, la incidencia de la pobreza entre 1984 y 1988 habría permanecido prácticamente igual. Ahora sabemos, por la ENIGH de 1989 (INEGI, 1992), que el ingreso entre 1984 y 1989 sufrió un proceso de concentración muy agudo (cuadro 2). La evaluación desde una medición de pobreza comparable con las anteriores del autor (lo cual supone entre otras cosas el ajuste a cuentas nacionales) es una tarea pendiente. Sin embargo, puesto que la distribución del ingreso empeoró, lós estratos intermedios -donde corta la línea de pobreza con el ingreso de los hogares- tienen un ingreso más bajo que lo que el autor supuso, por lo cral la pobreza será necesariamente más alta que la que él estimó, rebasando en 1989, 60\% de la población. Entre 1984 y 1989 el consumo privado per cápita -un indicador que refleja lo que ocurrió con el ingreso de los hogares-disminuyó en la economía en su conjunto a una tasa media anual de $-1.2 \%$. Si a esto añadimos el aumento en la concentración del ingreso: Gini de 0.43 al 0.47 (cuadro 2), veremos que el aumento de la pobreza tuvo que haber sido acelerado.

CUADRO 2

Coeficientes de Gini de la distribución del ingreso. Ingreso monetario y no monetario. 1984, 1989 y 1992

\begin{tabular}{lccc}
\hline Año & Ingreso total & Ingreso monetario & Ingreso no monetario \\
\hline 1984 & 0.4292 & 0.4562 & 0.5506 \\
1989 & 0.4694 & 0.4889 & 0.5921 \\
1992 & 0.4749 & 0.5086 & 0.5404 \\
\hline
\end{tabular}

Fuente: Cuadros 4, 5 y 6 de las eniGH, 1984 (tercer trimestre), 1989 y 1992. 
Aunque entre 1989 y 1992 -a diferencia del periodo anterior- el PIB creció más rápido que la población, la diferencia fue pequeña, por lo que el ingreso per cápita aumentó muy poco, y dado el deterioro en la distribución del ingreso monetario observado en esos años, la pobreza debió haber aumentado también, pero menos rápido que en los años anteriores.

Dado que el nivel de la pobreza en México estaba en 1984 en $58.5 \%$ (cuadro 1), la línea de pobreza cortaba la curva de ingresos de los hogares en el tramo superior del decil 6 de hogares. Por lo anterior, y dejando un margen amplio de un decil hacia arriba y uno hacia abajo, la clave para saber lo ocurrido entre 1989 y 1992 en materia de incidencia de la pobreza, consiste en determinar si el ingreso real de los deciles 5 , 6 y 7 continuó deteriorándose. Con datos de la ENIGH de 1989 y 1992 y sin ajustar a cuentas nacionales, analicé esta evolución en Boltvinik, 1994. Este análisis será válido en la medida en que ambas encuestas hayan subestimado el ingreso de estos deciles en la misma proporción, o que por lo menos la de 1992 no haya subestimado este ingreso en mayor medida que la de 1989. El ingreso total por hogar del decil 5 creció $2.1 \%$ en los tres años, el del decil $6,2.6 \%$ y el del decil $7,4.3 \%$. Sin embargo, observamos que el ingreso monetario por hogar de los tres deciles disminuyó en términos reales: $6.3 \%$ en el decil $5,3.1 \%$ en el 6, y $5.4 \%$ en el decil 7. En contraste, el ingreso no monetario por hogar en términos reales (deflactado por el índice general del índice nacional de precios al consumidor, INPC) creció en forma explosiva: $30.8 \%$ en el decil $5,22.4 \%$ en el 6, y $41.9 \%$ en el 7 . Es decir, es el ingreso no monetario el que crece y explica el crecimiento del ingreso total de estos deciles. Sin embargo, el ingreso no monetario presenta diversos problemas. Veamos cuáles son y qué correcciones se requieren para poder usarlos en el análisis de la evolución de la pobreza.

Observemos, en primer lugar, que el ingreso monetario representa entre tres cuartas y cuatro quintas partes del ingreso captado por las encuestas. El no monetario representó en 1984, 21.2\%, en $1989,22.5 \%$ y en $1992,26.1 \%$, mostrando que aunque es una parte minoritaria, su importancia ha venido creciendo, particularmente entre las dos últimas fechas. En segundo lugar, en las tres encuestas, cerca de las cuatro quintas partes del ingreso no monetario están compuestas por dos rubros: el alquiler imputado de la vivienda propia y los regalos en especie. Ambos rubros representan $78.7 \%$ en 1984 , $81.7 \%$ en 1989 y $83.7 \%$ en 1992 . Estos rubros aumentan de valor espectacularmente en el periodo: una tasa media anual de $5.6 \%$ entre 
1984 y 1989, y mucho más alta: 9.7\%, entre 1989 y 1992. Los dos rubros tienen particularidades que los hacen inadecuados para reflejar la evolución del nivel de vida real de las familias. El primero se refiere a los regalos recibidos solamente, cuando el concepto pertinente para un hogar individual es el concepto neto: regalos recibidos menos regalos otorgados. Es decir, a menos que hiciéramos la corrección -que sólo es posible si se trabaja con los registros de cada hogar-, el concepto es inservible.

A diferencia de los demás rubros de ingresos, el de alquiler imputado de la vivienda propia está constituido por un cálculo virtual de los "servicios prestados" por la vivienda a sus moradores. Si entre un año y otro hay un aumento en los precios relativos de la vivienda, quienes siguen viviendo en la misma casa (la inmensa mayoría en periodos cortos) aparecerán con ingresos mayores simplemente por efecto del cambio en los precios relativos. Incluso muchos hogares pueden dejar de ser pobres sólo por este efecto, aunque sus condiciones materiales de vida no se hayan modificado en lo más mínimo. Evidentemente algo de este tipo ocurrió entre 1989 y 1992, cuando el ingreso captado por la ENIGH en alquiler imputado aumentó $32.3 \%$ (24.2\% en términos per cápita). Este efecto desaparecería totalmente si los datos de 1992 fueran deflactados para expresarlos en pesos de 1989 usando índices de precios específicos.

Entre 1984 y 1992 el índice de precios de la vivienda aumentó 30.5\% más que el INPC. Entre 1984 y 1989 la tasa anual de crecimiento de los precios de la vivienda fue de $78.4 \%, 8 \%$ más rápida que la del nivel general de precios $(72.9 \%)$. Entre 1989 y 1992 la diferencia entre ambas tasas anuales fue aún mayor, de $19 \%: 28.3 \%$ la de vivienda y $23.7 \%$ la general.

Una corrección sencilla consiste en deflactar el alquiler imputado de la vivienda de 1992 con el índice de la vivienda en lugar del general. Ello basta para que el ingreso total por hogar en los deciles 5 y 6 prácticamente deje de crecer: 0.4 y $0.1 \%$; mientras que en el séptimo decil el incremento se reduce a $2.6 \%$. Si además se eliminan los ingresos por regalos -por lo antes apuntado- el ingreso total por hogar entre 1989 y 1992 en los deciles que venimos trabajando, cambia de signo y se reduce. Al aplicar las dos correcciones -la manera de deflactar la renta imputada y la eliminación de los regalos en ambos años-el ingreso total por hogar en los deciles 5, 6 y 7 se reduce entre 1989 y 1992 en 3.1, 2.7 y 1.6 por ciento.

Por tanto, con las reservas del caso, la pobreza entre 1989 y 1992 debió aumentar, aunque en una proporción pequeña, tal como se estima en el 
cuadro 1: de 64 a $66 \%$. Nos estamos acercando a los rangos de los sesenta. Por lo pronto, hemos rebasado con mucho los niveles de pobreza de 1977, haciendo que en materia de lucha contra la pobreza no sea una la década perdida, sino dos, y muy pronto tres. ${ }^{5}$ Los factores determinantes de esta evolución contrastante de la pobreza en ambos periodos son la evolución del empleo y las remuneraciones medias de éste, de lo cual no es inocente la política económica implantada, como puede verse en J. Boltvinik (1994).

\section{La magnitud de la pobreza en 1989}

\section{Incidencia de la pobreza y estratificación. MMIP}

De los 79 millones de personas que poblaban el país en 1989, 55.9 millones, que conformaban 10.2 millones de hogares y que representaban $70.6 \%$ de la población nacional, eran pobres según el MMIP, y 35.4

CUADRO 3

Estratificación e incidencia de la pobreza. MMrp nacional

\begin{tabular}{lccc}
\hline Estratos & Personas millones & Porcentaje del total & Porcentaje del subtotal \\
\hline Total de pobres & & & \\
$\quad$ extremos & 35.4 & 44.7 & 63.3 \\
$\quad$ Indigentes & 23.3 & 29.4 & 41.7 \\
$\quad$ Muy pobres & 12.1 & 15.3 & 21.7 \\
Pobres moderados & 20.5 & 25.9 & 36.7 \\
Total de pobres & 55.9 & 70.6 & 100.0 \\
& & & \\
Con Sanbri & 9.9 & 12.5 & 42.5 \\
Clase media & 9.0 & 11.4 & 38.6 \\
Clase alta & 4.4 & 5.5 & 18.9 \\
Total de no pobres & 23.3 & 29.4 & 100.0 \\
Total & 79.1 & 100.0 & \\
\end{tabular}

Fuente: en éste como en los restantes cuadros es elaboración propia.

5 Un grupo de estudiantes del rTAM calculó la incidencia de la pobreza en México en 1992 - con base en los datos publicados de la ENIGH en 1992-, siguiendo la metodología de la cNSE y realizando el ajuste a cuentas nacionales con el mismo procedimiento aplicado a las encuestas de 1963 a 1984 por Hernández Laos. El resultado es una incidencia de $65.2 \%$, que confirma el cálculo hipotético presentado en el cuadro 1 . Véase Jana Boltvinik et al. (1994). 
millones (44.7\%) eran pobres extremos. Al descomponer el grupo de los pobres extremos en los dos estratos que lo conforman, observamos que los indigentes -los peor situados-representaban $29.4 \%$ de la población nacional (23.3 millones) y los muy pobres $15.3 \%$ (12.1 millones). ${ }^{6}$ Quedan entonces como pobres moderados 20.5 millones, es decir, $25.9 \%$ de la población nacional (cuadro 3).

Los 23.3 millones de personas no pobres (29.4\% de la población) han sido clasificados en tres estratos de la siguiente manera: con satisfacción de necesidades básicas y de requerimientos de ingresos (Sanbri), 9.9 millones ( $12.5 \%$ de la población); en la clase media, 9.0 millones ( $11.4 \%$ ), y en la clase alta 4.4 millones, o sea, $5.5 \%$ de los habitantes del país (cuadro 3 ). ${ }^{7}$

La incidencia de la pobreza es significativamente más alta en el campo que en las ciudades: $85 \%$ vs. $61.7 \%$. Si se consideran los porcentajes de no pobres, se apreciará la enormidad del contraste: $38.3 \%$ en el sector urbano y sólo $15 \%$ en el rural, es decir, 2.55 veces más en el primero que en el segundo (cuadro 4).

CUADRO 4

Estratificación e incidencia de la pobreza (н) por tipo de localidad. MarP Porcentajes de la población total de cada contexto geográfico $(\mathrm{H})$

\begin{tabular}{lccrrrr}
\hline Estratos del Mmr & Nacional & $\begin{array}{c}\text { Urbano } \\
\text { Total }\end{array}$ & zMCM & $\begin{array}{c}\text { Metropo- } \\
\text { litana }\end{array}$ & $\begin{array}{c}\text { No Metro- } \\
\text { politana }\end{array}$ & Rural \\
\hline Pobres extremos & 44.7 & 34.1 & 33.1 & 29.5 & 37.2 & 61.8 \\
$\quad$ Indigentes & 29.4 & 19.3 & 17.0 & 14.9 & 23.4 & 45.7 \\
$\quad$ Muy pobres & 15.3 & 14.8 & 16.1 & 14.6 & 13.9 & 16.1 \\
Pobres moderados & 25.9 & 27.6 & 28.3 & 29.1 & 26.3 & 23.2 \\
Total pobres & 70.6 & 61.7 & 61.4 & 58.6 & 63.6 & 85.0 \\
Con Sanbri & 12.5 & 15.8 & 14.9 & 17.9 & 15.3 & 7.2 \\
Clase media & 11.4 & 15.0 & 15.3 & 16.2 & 14.1 & 5.6 \\
Clase alta & 5.5 & 7.6 & 8.5 & 7.3 & 7.0 & 2.2 \\
Total no pobres & 29.4 & 38.3 & 38.6 & 41.4 & 36.4 & 15.0 \\
Población total & 100.0 & 100.0 & 100.0 & 100.0 & 100.0 & 100.0 \\
\hline
\end{tabular}

6 Conviene notar que quedan clasificados como indigentes los hogares y personas que, en promedio, satisfacen menos de la mitad del conjunto de normas mínimas definidas por los dos métodos parciales; los muy pobres son los que satisfacen entre la mitad y menos de dos terceras partes de las normas, y los pobres moderados son los que satisfacen entre dos tercios y menos de $95 \%$ de las normas. A partir de este último nivel no se consideran pobres.

${ }^{7}$ Los estratos se conformaron clasificando dentro del estrato que cuenta con satisfacción de necesidades básicas y de requerimientos de ingresos a los que tienen una 
Un resultado sorpresivo es que la pobreza en las metrópolis (áreas urbanas mayores de 500000 habitantes) es más baja que en la zмcM $(58.6 \%$ vs. $61.4 \%)$, mientras que las ciudades menores de 500000 habitantes muestran -de acuerdo con lo esperado-, una incidencia global más alta: 63.6 por ciento.

Las diferencias urbano-rurales se hacen mayores si tomamos en cuenta que $61.8 \%$ de los habitantes del campo es pobre extremo (suma de indigentes y muy pobres), mientras el porcentaje urbano representa un poco más de la mitad de aquél: $34.1 \%$. En la proporción de indigentes el contraste es todavía más fuerte: en el medio rural $45.7 \%$ se encuentra en esta situación, mientras que en las ciudades la cifra correspondiente es 2.5 veces más baja: $19.3 \%$. En el otro extremo de la escala social, los contrastes son todavía más agudos. En la clase media el porcentaje urbano es 2.7 veces mayor que el rural: $15.0 \%$ vs. $5.6 \%$, y en la clase alta la proporción es 3.5 veces mayor: $7.6 \%$ vs. $2.2 \%$ (cuadro 4 ).

Mientras en el medio rural (baja densidad) habita $38.2 \%$ de la población del país, vive en él $46.0 \%$ de los pobres. En contraste, el medio urbano (alta densidad) participa en la población nacional con $61.8 \%$ y en el total de pobres con $54.0 \%$. Las diferencias se acentúan cuando se analizan los extremos de la pirámide social: habita en el medio rural $59.4 \%$ de los indigentes y $52.8 \%$ de los pobres extremos, y sólo $15.3 \%$ de la clase alta y $18.8 \%$ de la clase media (cuadro 5 ).

En las áreas urbanas mayores, que constituyen el contexto geográfico mejor situado, vive $14.4 \%$ de la población nacional, $12.0 \%$ de los pobres, sólo $7.3 \%$ de los indigentes y $20.5 \%$ de la clase media. La zмсм participa en la población con $20.1 \%$, más que proporcionalmente en la clase alta con $30.8 \%$, y menos que proporcionalmente entre los indigentes: $11.6 \%$. Sin embargo, como consecuencia de participaciones más que proporcionales entre los muy pobres y los pobres moderados $(21.2$ y $21.9 \%)$, su proporción en el total de los pobres no queda

I(MMr) entre 0.05 y -0.09 , es decir, alrededor de la norma, en la clase media cuando dicho indicador vale entre -0.1 y -0.49 y, por último, en la clase alta cuando el valor es -0.5 o menor. La simetría entre este límite y el de los indigentes $(0.5)$ debe interpretarse en el siguiente sentido: la clase alta comienza a partir de la mitad entre el valor de las normas y el valor máximo usado para la reescalación, y que se puede identificar conceptualmente como el punto arriba del cual no es factible aumentar el bienestar en la dimensión específica. De esta manera, el requisito mínimo de la clase alta es que se encuentre al menos a la mitad entre la norma y ese punto de máxima satisfacción. Los indigentes -simétricamente- están entre la mitad de la norma y la máxima insatisfacción posible. 
muy por debajo de su participación en la población total: $17.5 \%$ (cuadro 5).

CUADRO 5

Distribución geográfica de la población de cada estrato por tipo de localidad. MMIP (porcentaje del total nacional del estrato)

\begin{tabular}{|c|c|c|c|c|c|c|}
\hline Estratos del ${ }_{M M P P}$ & Nacional & $\begin{array}{c}\text { Total } \\
\text { urbano }\end{array}$ & ZMCM & $\begin{array}{l}\text { Urbano } \\
\text { metrop. }\end{array}$ & $\begin{array}{l}\text { Urb. no } \\
\text { metrop. }\end{array}$ & $\begin{array}{l}\text { Total } \\
\text { rural }\end{array}$ \\
\hline Pobres extremos & 100.00 & 47.2 & 14.9 & 9.5 & 22.8 & 52.8 \\
\hline Indigentes & 100.00 & 40.6 & 11.6 & 7.3 & 21.7 & 59.4 \\
\hline Muy pobres & 100.00 & 59.7 & 21.2 & 13.8 & 24.7 & 40.3 \\
\hline Pobres moderados & 100.00 & 65.9 & 21.9 & 16.2 & 27.8 & 34.1 \\
\hline Total de pobres & 100.00 & 54.0 & 17.5 & 12.0 & 24.5 & 46.0 \\
\hline Con Sanbri & 100.00 & 78.0 & 23.9 & 20.6 & 33.5 & 22.0 \\
\hline Clase media & 100.00 & 81.2 & 27.0 & 20.5 & 33.7 & 18.8 \\
\hline Clase alta & 100.00 & 84.7 & 30.8 & 19.0 & 34.9 & 15.3 \\
\hline No pobres & 100.00 & 80.5 & 26.4 & 20.3 & 33.8 & 19.5 \\
\hline Población total & 100.00 & 61.8 & 20.1 & 14.4 & 27.3 & 38.2 \\
\hline
\end{tabular}

Incidencia de la pobreza y estratificación por los métodos parciales y por la versión matricial del MMIP

El porcentaje de población pobre por NBI, tanto en el ámbito nacional como en el urbano y rural, es sustancialmente más alto que el de LPT. La diferencia para el país en su conjunto es de casi 18 puntos porcentuales: 76.8 vs. $58.9 \%$ (cuadro 6 ). En términos de número de personas esta diferencia es de 14.2 millones: 60.8 contra 46.6 millones.

Los pobres extremos por NBI representan $71.6 \%$ de los pobres considerados por ese método, y la proporción respectiva por LPT es de $70.1 \%$ que, como se ve, es muy similar. La proporción de indigentes es también ligeramente mayor por NBI que por LPT: 50.4 vs. 49.6 por ciento (cuadro 6).

Con el Mмip "matricial" los pobres resultan ser $80.6 \%$ de la población nacional, 10 puntos porcentuales más que si se aplica el MMIP "promedial". 8 De la población, $55.1 \%$ es pobre por ambos métodos parciales (LPT y NBI), es decir, padece simultáneamente las carencias

8 En la versión matricia al del Mмı se definen como pobres los hogares que resultan así clasificados en uno de los dos métodos parciales. Por tanto, como se aprecia en el 
CUADRO 6

Principales parámetros por tipo de localidad

\begin{tabular}{lcccccc}
\hline $\begin{array}{l}\text { Tipo de localidad } \\
\text { (porcentaje*) }\end{array}$ & Nacional & Urbano & Rural & $\begin{array}{c}\text { Urbano } \\
>500 \text { mil }\end{array}$ & $\begin{array}{c}\text { Urbano } \\
<500 \text { mil }\end{array}$ & zMCM \\
\hline H(NBI)* & 76.8 & 67.7 & 91.6 & 64.8 & 69.5 & 67.1 \\
H(LPT) \% & 58.9 & 51.1 & 71.4 & 47.2 & 53.5 & 50.7 \\
Pobext/pob NBI* & 71.6 & 60.4 & 85.1 & 58.2 & 64.6 & 55.9 \\
Pobext/pob LPT* & 70.1 & 65.4 & 75.6 & 61.7 & 68.2 & 63.9 \\
Indig/pob NB̈* & 50.4 & 34.8 & 69.0 & 28.8 & 41.7 & 29.4 \\
Indig/pob LPT* & 49.6 & 42.5 & 57.9 & 39.0 & 45.1 & 41.0 \\
H(MMPMatr) & 80.6 & 73.0 & 93.0 & 70.5 & 74.7 & 72.4 \\
H(PobTot)* & 55.1 & 45.8 & 70.1 & 41.5 & 48.4 & 45.4 \\
H(PobextTot) & 32.4 & 22.2 & 49.0 & 17.0 & 26.7 & 19.7 \\
H(IndigTotal) & 19.3 & 10.5 & 33.6 & 6.8 & 13.8 & 8.6 \\
H(sólo NBI) & 21.7 & 21.8 & 21.6 & 23.3 & 21.2 & 21.7 \\
H(sólo LPT)* & 3.8 & 5.3 & 1.4 & 5.7 & 5.2 & 5.3 \\
H(MMIP)* & 70.6 & 61.7 & 85.0 & 58.6 & 63.6 & 61.4 \\
I(MMIP) & 0.44 & 0.39 & 0.50 & 0.35 & 0.41 & 0.37 \\
I(NBI) pobres MMIP & 0.51 & 0.42 & 0.61 & 0.39 & 0.46 & 0.39 \\
I(LPT) pobres MMIP & 0.40 & 0.36 & 0.43 & 0.33 & 0.39 & 0.35 \\
I(NBI) pobres NBI & 0.50 & 0.42 & 0.59 & 0.39 & 0.45 & 0.39 \\
I(LPT) pobres LPI & 0.49 & 0.45 & 0.53 & 0.42 & 0.47 & 0.44 \\
HI(MMIP) & 0.311 & 0.241 & 0.425 & 0.205 & 0.261 & 0.227 \\
HI(NBI) & 0.38 & 0.28 & 0.54 & 0.25 & 0.31 & 0.26 \\
HI(LPT) & 0.29 & 0.23 & 0.38 & 0.20 & 0.25 & 0.22 \\
\hline
\end{tabular}

que se detectan por ambos procedimientos. Constituye el universo de pobres "totales" o, como les llamara Rubén Kaztman, pobres crónicos. Existe una gran asimetría en cuanto a las pobrezas parciales. Una proporción muy importante de la población es pobre sólo por NBI: $21.7 \%$, mientras que la pobreza sólo por LPT es casi una rareza: 3.8\% (cuadro 7).

$\mathrm{Al}$ igual que en el ámbito nacional, en el medio rural la incidencia por LPT es menor que por NBI: 71.4 vs. $91.6 \%$, una diferencia de más de 20 puntos porcentuales. En el medio urbano la pobreza por LPT es de $51.1 \%$, mientras la de NBI la supera en casi 17 puntos porcentuales: $67.7 \%$. Como se aprecia, en ambos contextos la diferencia es

cuadro 7 , los pobres son la suma de tres celdas de la tabla de contingencia: los pobres por ambos métodos, los pobres sólo por NBI y los pobres sólo por LPT. 
CUADRO 7

Matriz de la pobreza por LPT y Nы

\begin{tabular}{|c|c|c|c|}
\hline & $\begin{array}{c}\text { LPT } \\
\text { Pobres } \\
\text { (porcentajes) }\end{array}$ & $\begin{array}{c}L P T \\
\text { No pobres } \\
\text { (porcentajes) }\end{array}$ & $\begin{array}{c}\text { Suma NBI } \\
\text { (porcentajes) }\end{array}$ \\
\hline NBI pobres & 55.1 & 21.7 & 76.8 \\
\hline NBI no pobres & 3.8 & 19.4 & 23.2 \\
\hline Sumas LP & 58.9 & 41.1 & 100.0 \\
\hline \multicolumn{4}{|c|}{$\begin{array}{l}\text { Suma pobres MMIP matricial: H(MMIPmatr): } 86 \\
\text { Pobres totales o crónicos: H(PobTot): } 55.1 \\
\text { Pobres sólo por NBI: H(sólo NBI): } 21.7 \\
\text { Pobres sólo por LPT: H(sólo LPT): } 3.8 \\
\text { No pobres MMIP matricial: } 19.4\end{array}$} \\
\hline
\end{tabular}

CUADROS $7 \mathrm{a}$ y $7 \mathrm{~b}$

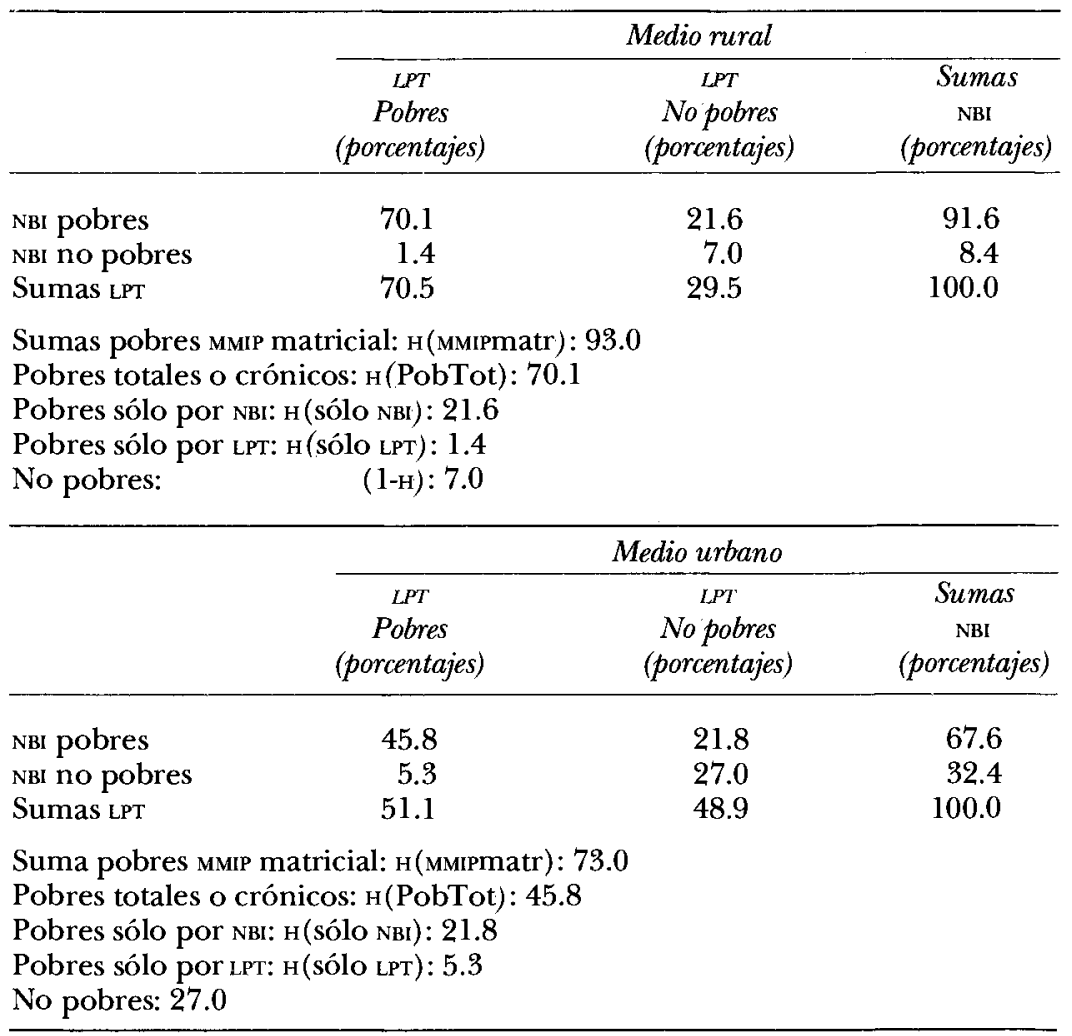


similar. En el medio rural la inmensa mayoría de los pobres por NBI (85.1\%) está integrada por pobres extremos y más de dos terceras partes son indigentes $(69 \%)$. Este grado de concentración tan alto en los niveles más agudos de la pobreza no se presenta en la pobreza por ingresos-tiempo, ni en el medio urbano. Los pobres extremos por LPT en el medio rural representan tres cuartas partes de los pobres rurales, y los indigentes $57.9 \%$ (cuadro 6).

En las ciudades, la proporción de pobres extremos es más baja en ambas dimensiones que en el campo, pero además es más alta por LPT $(65.4 \%)$ que por NBI $(60.4 \%)$-contrariamente a lo que sucede en el ámbito rural. Lo mismo ocurre respecto de los indigentes urbanos, que representan $42.5 \%$ de los pobres por LPT y $34.8 \%$ por NBr. Sin embargo, dada la mayor incidencia de la pobreza por NBr, el número absoluto de pobres extremos e indigentes urbanos es más alto por NBI que por LPT: 20 millones y 11.5 millones $v s$. 16.4 millones y 10.6 millones, respectivamente.

La pobreza que el MMIP matricial identifica es $93 \%$ en el medio rural y $73 \%$ en el urbano. En el medio urbano, $62.7 \%$ es pobre total, mientras que en el rural llega a $75.4 \%$. El contraste en la proporción de no pobres es enorme: 7 vs. 27\%, casi cuatro veces. En ambos medios poco más de la quinta parte es pobre sólo por NBI (cuadro 7).

Los resultados de incidencia de la pobreza obtenidos hasta ahora muestran que de los diferentes cálculos el más alto está dado por el MMIP matricial (o LPT-NBI), $80.6 \%$; el método de NBI ocupa el segundo lugar con $76.8 \%$, en tercero el MMIP con $70.6 \% \mathrm{y}$, por último, con la estimación más baja, el de LPT, con 58.9\% (cuadro 8).

CUADRO 8

Incidencias de la pobreza (н) con los diferentes métodos

\begin{tabular}{lcccc}
\hline & $M M I P$ & $N B I$ & $L P T$ & $L P N B I$ \\
\hline Nacional & & & & \\
$\quad$ Pobres & 70.6 & 76.8 & 58.9 & 80.6 \\
$\quad$ No pobres & 29.4 & 23.2 & 41.1 & 19.4 \\
Rural & & & & \\
$\quad$ Pobres & 85.0 & 91.6 & 71.4 & 93.0 \\
$\quad$ No pobres & 15.0 & 8.4 & 28.6 & 7.0 \\
Urbano & & & & \\
$\quad$ Pobres & 61.7 & 67.6 & 51.1 & 73.0 \\
$\quad$ No pobres & 38.3 & 32.4 & 48.9 & 27.0 \\
\hline
\end{tabular}


La incidencia de la pobreza por NBI es más alta que la de LPT en todos los contextos geográficos. Las ciudades de más de 500000 habitantes tienen una mejor situación que la zмсм en ambas dimensiones y prácticamente en todos los parámetros, y ambas están -a su vez- mejor que las ciudades de menos de 500000 habitantes.

Los individuos que son pobres, pobres extremos o indigentes en ambas dimensiones, se han denominado pobres totales, pobres extremos totales e indigentes totales. En el ámbito nacional $55.1 \%$ de la población vive en pobreza total $-\mathrm{H}$ (PobTot)-, 32.4\% en pobreza extrema total $-\mathrm{H}($ PobExtTot $)-$ y $19.3 \%$ en indigencia total $-\mathrm{H}$ (IndigTot). Estas proporciones son más bajas en las áreas urbanas y más altas en las rurales. Los espacios mejor situados, las zonas urbanas de más de 500000 habitantes, tienen en las mismas categorías $41.5,17.0$ y $6.8 \%$, mientras las rurales tienen, respectivamente, $70.1,49.0$ y $33.6 \%$. Como se ve, el contraste más fuerte se da en la indigencia total, donde el dato rural es casi cinco veces más grande que el metropolitano, mientras en la pobreza total el contraste es de 1.7 a 1 (cuadro 6).

\section{Intensidad de la pobreza}

La intensidad de la pobreza para el conjunto de los pobres conforme al MMIP (0.44) resulta de la combinación entre las dos intensidades parciales, de las cuales es bastante más alta la de NBI que la de LPT: 0.51 vs. 0.40. Esta diferencia radica en que la I(LPT) de los pobres moderados por el Mmip es muy cercana a la norma: $(0.08)$, en agudo contraste con I(NBI) del mismo estrato, que es muy alta: casi cinco veces más (0.38). Entre los muy pobres la diferencia entre ambas intensidades parciales es mucho menor y en los indigentes la situación se invierte, siendo la I(LPT) la más alta ( 0.67 vs. 0.64$)$. Para el conjunto de los pobres extremos, la intensidad de la pobreza es igual por ambos métodos: 0.58 (cuadro 8a).

La pregunta que el análisis de i intenta responder es ¿qué tan pobres son los pobres? La respuesta sintética significa que, en promedio, los pobres del país tienen una brecha de $44 \%$ respecto al conjunto de las normas, o de otro modo, que sólo cumplen con $56 \%$ de ellas. Nuestro pobre promedio sería considerado como muy pobre y, dentro de este estrato, más cerca del indigente que del moderado. Su valor promedio en $\mathrm{I}(\mathrm{NBI}): 0.51$, lo clasificaría como indigente, mientras que su indicador de LPT $(0.40)$ lo ubicaría entre los muy pobres. 


\section{CUADRO 8a}

Valores medios de los indicadores de carencia por estratos del MMIP. Total nacional

\begin{tabular}{lcccc}
\hline Estratos del MMIP & $\begin{array}{c}H \\
\text { (porcentaje) }\end{array}$ & $I($ MIP $)$ & $I(\mathrm{NHt})$ & $I(L P T)$ \\
\hline Pobres extremos & 44.7 & 0.58 & 0.58 & 0.58 \\
$\quad$ Indigentes & 29.4 & 0.66 & 0.64 & 0.67 \\
$\quad$ Muy pobres & 15.3 & 0.42 & 0.46 & 0.40 \\
Pobres moderados & 25.9 & 0.19 & 0.38 & 0.08 \\
Total de pobres & 70.6 & 0.44 & 0.51 & 0.40 \\
Con Sanbri & 12.5 & -0.02 & 0.11 & -0.10 \\
Clase media & 11.4 & -0.26 & -0.06 & -0.37 \\
Clase alta & 5.5 & -0.68 & -0.26 & -0.93 \\
Total de no pobres & 29.4 & -0.24 & -0.03 & -0.36 \\
Población total & 100.0 & 0.24 & 0.35 & 0.17 \\
& & & & \\
\hline
\end{tabular}

Los indigentes por el MMIP tienen una I(LPT) de 0.67, que significa que su ingreso - una vez ajustado, en su caso, por el exceso de traba:jo- es la tercera parte del ingreso de la línea de pobreza, y menos de la mitad $(46.5 \%)$ de la línea de pobreza extrema, lo que refleja una situación insostenible incluso desde el punto de vista nutricional. Lamentablemente, en esta situación se encuentra $29.4 \%$ de los habitantes del país. La condición de vida de los indigentes, que se captura sintéticamente con una I(MMIP) de 0.67 es, sin duda, una situación de miseria abismal (cuadro 8a).

Los muy pobres son un grupo de menor tamaño ( $15.3 \%$ de la población nacional) y con rangos de intensidad de la pobreza que están sólo un poco abajo de 0.5 . En ingresos-tiempo cubren $60 \%$ de los requerimientos, por debajo de la línea de pobreza extrema, pero muy cerca de ella. En NBI están muy cerca de la mitad de las normas, a sólo cuatro centésimas de la indigencia en la dimensión (cuadro 8a).

Conjuntamente, los muy pobres y los indigentes constituyen el grupo de los pobres extremos, que representa $44.7 \%$ de la población nacional y cuya situación promedio es dramática: dejan de cubrir casi $60 \%$ de sus requerimientos de ingresos y de satisfactores específicos $(58 \%)$. Se trata de cerca de la mitad de la población, que vive con dos quintas partes de los satisfactores básicos. 
Los pobres moderados, un poco más de la cuarta parte de la población nacional (25.9\%), logran ingresos muy cercanos a la línea de pobreza, pero tienen carencias significativas por NBI (que los calificarían como muy pobres en esa dimensión).

Los no pobres muestran, coherentemente, indicadores de carencia negativos. El grupo en su conjunto tiene una I(MмIP) de -0.24 , que resulta de -0.03 en I(NBI) y de -0.36 en I(LPT), denotando la segunda una carencia negativa doce veces menor, o bienestar doce veces mayor, que la primera.

La intensidad de la pobreza es sustancialmente más alta en el medio rural que en el urbano con cualquiera de nuestras dos medidas parciales y con la integrada. El contraste más agudo se presenta en NBI: la I(NBI) rural (del conjunto de pobres identificados por el MMIP) es casi $50 \%$ más alta que la urbana: 0.61 vs. 0.42 ; mientras la diferencia en las intensidades de la pobreza por LPT, es mucho menor, alrededor de 20\%: 0.43 vs. 0.36. Como resultado I(ммір) es 0.5 en el medio rural y 0.39 en el urbano, una diferencia de $28 \%$ (cuadro 6 ).

En cuanto al comportamiento de I(MMIP) por tamaño de localidades, observamos el mismo patrón que habíamos encontrado para н(мміP) : la intensidad más baja se presenta en las metrópolis de más de 500000 habitantes, 0.35 , seguida por la zмсм con 0.37 y por las ciudades menores con 0.41 . En el medio rural, como ya se había señalado, llega a 0.50 (cuadro 6).

\section{Descomposición de los indices de NBI y de LPT. Perfil de carencias}

Sinteticemos el procedimiento para obtener el I(NBI). Se trata de una media ponderada de los siguientes indicadores de carencia. Su ponderador, que se basa en costos, se indica al final entre paréntesis:

CCEV: carencia en la calidad y espacios de la vivienda. Indicador que a su vez es el producto del indicador de carencia en la calidad (materiales de construcción) de la vivienda ( $\mathrm{CCv}$ ), y el de hacinamiento multidimensional (HMD). Su ponderador es el más alto $(0.325)$.

cs: carencia sanitaria (agua, drenaje y baño) (0.037).

Cots: carencia en otros servicios (electricidad y teléfono) (0.06).

CBD: carencia de bienes durables o indicador de patrimonio (0.058).

$\mathrm{RE}$ : rezago educativo del hogar (alfabetismo y grado de instrucción) (0.237). 
CAss: carencia en atención a la salud y seguridad social (0.282).

Los ponderadores utilizados fueron obtenidos del costo que, en junio de 1989, representaban estos rubros, el cual se obtuvo actualizando los costos de la cNSE elaborada en Coplamar. Los costos reflejan los precios de mercado, ya que son éstos los que la población debe cubrir para adquirir los bienes.

En el medio mural, la intensidad de la pobreza por NBI, denotada en el cuadro 9 como I, para el conjunto de los pobres identificados por este procedimiento, es 0.59 que califica al pobre rural promedio como indigente, y los tres indicadores sectoriales de carencia con ponderadores más altos tienen los siguientes valores: CCEv: 0.65 ; $\mathrm{RE}: 0.47, \mathrm{y}$ cass: $0.73 .{ }^{9}$ En los indicadores de menor ponderación los valores promedio para los pobres rurales son: cs (servicios sanitarios): 0.65; Cots (electricidad): 0.14 y CBD (patrimonio básico): 0.48 . Salvo en la electricidad, donde la carencia es baja, el rango de los demás indicadores está entre 0.47 (educación), como la menos intensa de las carencias y 0.73 , acceso a la salud, como la más intensa. Estos contrastes muestran la heterogeneidad en la (in) satisfacción de las necesidades. Salvo en el caso de la electricidad, todas las necesidades se encuentran en el rango de carencia aguda -CCEV, CASs y cs en el nivel de indigencia, $R E$ y CBD en el de muy pobres, y el valor medio resultante, $\mathrm{I}(\mathrm{NBI})$, también en el de indigencia.

Para los pobres por NBI en el medio urbano el valor promedio de $\mathrm{I}(\mathrm{NBI})$ es de $0.42,17$ puntos menos que en el rural, que haría calificar al pobre promedio urbano por NBI como muy pobre en esta dimensión. Los tres componentes de gran peso arrojan los valores siguientes: cCEv, $0.52,13$ centésimas menos que en el medio rural, pero 10 centésimas por arriba de su propia $\mathrm{I}(\mathrm{NBI})$ media y en un nivel de indigencia; $\mathrm{RE}$, 0.24, 23 centésimas menos que en el medio rural -prácticamente la mitad- y muy por abajo de la I(NBI), en un rango de pobreza moderada; cass, el indicador de acceso a la salud está, en cambio, muy por arriba del promedio -en plena indigencia-y solamente trece centésimas abajo del valor del medio rural: 0.60 . Los valores de los otros tres indicadores de menor peso son: cs: 0.17 , un poco más de la cuarta parte del 0.65 del medio rural y muy por abajo de su propia media, en el

${ }^{9}$ No se analiza en este artículo, por falta de espacio, el índice que se denomina $\mathrm{HI}$ y que puede interpretarse como magnitud de la pobreza: el producto de la proporción de pobres por la intensidad de la pobreza. En el cuadro 6, sin embargo, el lector puede consultar los principales resultados alcanzados. 
CUADRO 9

Descomposición del índice de I(NBI).

Medios urbano y rural. Alternativas de ponderación

\begin{tabular}{|c|c|c|c|c|c|c|c|c|c|}
\hline \multicolumn{10}{|c|}{ Área rural } \\
\hline Estratos/NBI & $I$ & CCEV & $C C V$ & $H M D$ & $C S$ & Cots & $C B D$ & $R E$ & CASS \\
\hline Pob Ext & 0.65 & 0.70 & 0.45 & 0.49 & 0.70 & 0.16 & 0.56 & 0.51 & 0.83 \\
\hline Indigentes & 0.71 & 0.75 & 0.48 & 0.54 & 0.74 & 0.18 & 0.64 & 0.54 & 0.92 \\
\hline Muy Pọb & 0.42 & 0.50 & 0.29 & 0.32 & 0.53 & 0.07 & 0.22 & 0.39 & 0.47 \\
\hline Pob Mod & 0.24 & 0.36 & 0.21 & 0.19 & 0.38 & 0.02 & 0.00 & 0.24 & 0.17 \\
\hline Sum Pob & 0.59 & 0.65 & 0.41 & 0.45 & 0.65 & 0.14 & 0.48 & 0.47 & 0.73 \\
\hline C. $/$ nbs & 0.00 & 0.13 & 0.13 & -0.01 & 0.27 & -0.01 & -0.18 & 0.09 & -0.22 \\
\hline Cla Med & -0.22 & -0.16 & 0.13 & -0.33 & 0.17 & -0.01 & -0.42 & 0.03 & -0.57 \\
\hline Cla Alt & -0.55 & -0.51 & 0.01 & -0.52 & 0.00 & -0.02 & -0.32 & -0.40 & -0.95 \\
\hline No Pob & -0.08 & 0.03 & 0.13 & -0.12 & 0.23 & -0.01 & -0.25 & 0.05 & -0.34 \\
\hline Pobrur & 0.53 & 0.60 & 0.39 & 0.40 & 0.62 & 0.12 & 0.42 & 0.43 & 0.64 \\
\hline \multicolumn{10}{|c|}{ Área urbana total } \\
\hline Estratos/NBI & $I$ & CCEV & $\mathrm{CCV}$ & $H M D$ & $C S$ & Cots & $C B D$ & $R E$ & CASS \\
\hline Pob Ext & 0.55 & 0.62 & 0.25 & 0.51 & 0.24 & 0.26 & 0.21 & 0.33 & 0.82 \\
\hline Indigentes & 0.64 & 0.71 & 0.30 & 0.60 & 0.31 & 0.27 & 0.35 & 0.42 & 0.94 \\
\hline Muy Pob & 0.42 & 0.50 & 0.18 & 0.40 & 0.14 & 0.26 & 0.01 & 0.21 & 0.66 \\
\hline Pob Mod & 0.22 & 0.37 & 0.11 & 0.29 & 0.08 & 0.21 & -0.15 & 0.10 & 0.25 \\
\hline Sum Pob & 0.42 & 0.52 & 0.20 & 0.42 & 0.17 & 0.24 & 0.07 & 0.24 & 0.60 \\
\hline C/nbs & 0.00 & 0.11 & 0.06 & 0.05 & 0.04 & 0.10 & -0.41 & -0.06 & 0.00 \\
\hline Cla Med & -0.26 & -0.11 & 0.04 & -0.16 & 0.01 & 0.03 & -0.64 & -0.25 & -0.45 \\
\hline Cla Alt & -0.61 & -0.55 & 0.01 & -0.57 & 0.00 & 0.01 & -0.85 & -0.50 & -0.94 \\
\hline No Pob & -0.15 & -0.03 & 0.05 & -0.08 & 0.03 & 0.07 & -0.53 & -0.17 & -0.25 \\
\hline Total & 0.24 & 0.34 & 0.15 & 0.26 & 0.13 & 0.19 & -0.13 & 0.11 & 0.32 \\
\hline
\end{tabular}

Nota: I-1 es la I(NBI) tal como se calculó de acuerdo con el anexo metodológico hogar por hogar 1-2, e I-3 se calculan de acuerdo con las siguientes ecuaciones que se aplican a los valores medios de los estratos: $1-2=(\mathrm{CCV}+\mathrm{HMD}+\mathrm{Cs}) 0.125+(\mathrm{Cots}+\mathrm{CBD}) 0.0625$ $+(\mathrm{RE}) 0.25+(\mathrm{CASS}) 0.25 .1-3=(\mathrm{CCV}+\mathrm{HMD}) 0.1625+(\mathrm{CS}) .037+(\mathrm{Cots}) 0.06+(\mathrm{CBD}) 0.058$ $+(\mathrm{RE}) 0.25+\mathrm{CASs}(0.25)$.

rango de pobreza moderada; Cots, que aquí incluye teléfono privado para las ciudades de más de 500000 habitantes, tiene un valor de 0.24, muy por arriba del rural, por la diferencia en el requerimiento, pero todavía en el rango de pobreza moderada; $\mathrm{CBD}$, el indicador de patrimonio durable, coloca al promedio de hogares pobres casi en la norma, con un sorprendente 0.07 , que correspondería al estrato global de NBs (cuadro 9). 
La variabilidad de los indicadores medios del conjunto de los pobres es mayor en el medio urbano que en el rural. Incluso si quitamos el caso más desviado, como hicimos en el medio rural con otros servicios, el rango restante va desde 0.17 hasta 0.60 : 43 puntos, mientras en el medio rural es de sólo 26 puntos. Pero además la variabilidad entre los tres indicadores que han recibido las ponderaciones altas es también mayor en el medio urbano: desde 0.24 en educación hasta 0.60 en acceso a salud, mientras que en el rural la variación es de 0.47 en educación, a 0.73 en salud ( 36 puntos contra 26). Esto permite sostener que en el medio urbano el índice es más sensible a los ponderadores utilizados.

Analicemos, por último, la integración de los indicadores de ingresos y tiempo. La lógica de la combinación se deriva, en primer lugar, de la inclusión del tiempo libre o disponible como una de las fuentes de bienestar del hogar. En segundo lugar, se interpreta el tiempo de trabajo como el complemento del libre, y la presencia de sobretrabajo (subtrabajo) se considera como un posible síntoma de insuficiencia (excedente) de la fuente de bienestar-tiempo libre. Sólo se califica la combinación ingresos-tiempo. Lo que se hace es corregir el ingreso del hogar para obtener el que tendría si su tiempo de trabajo se ajustara a la norma, considerándose la corrección por subtrabajo sólo cuando el ingreso es mayor que la línea de pobreza (se adopta el supuesto de que el subtrabajo de los pobres es involuntario). La corrección por sobretrabajo, en cambio, se lleva a cabo en todos los casos. En la definición de la norma de trabajo extradoméstico se consideran los requerimientos variables por hogar de trabajo doméstico y estudio. cy es el indicador de carencia por ingresos antes de tomar en cuenta el tiempo. ET indica sobretrabajo cuando vale más de 1.0 y subtrabajo cuando vale menos de 1.0. El cuadro 10 sintetiza los datos de los indicadores cy y ET. Además, compara los resultados de incidencia por estratos entre LP y LPT.

Al combinar la dimensión de ingresos con la de tiempo de trabajo (ET), la incidencia de la pobreza pasa de $56.6 \%$ con ingresos solamente [H(LP) ], a $58.9 \%$ con ingresos-tiempo $\mathrm{H}(\mathrm{LPT})$. El aumento ocurre tanto en el medio urbano como en el rural, pero en mayor medida en el primero, donde aumenta 2.7 puntos porcentuales $v s$. 1.7 puntos en el segundo. Por estratos, todo el incremento ocurre entre los indigentes, que aumentan de 26.9 a $29.2 \%$ de la población nacional.

ET no sólo modifica la estructura de la estratificación, la forma de la pirámide social, sino que afecta también la intensidad de la pobreza o del bienestar. Si a $56.6 \%$ de la población nacional considerada como pobre 


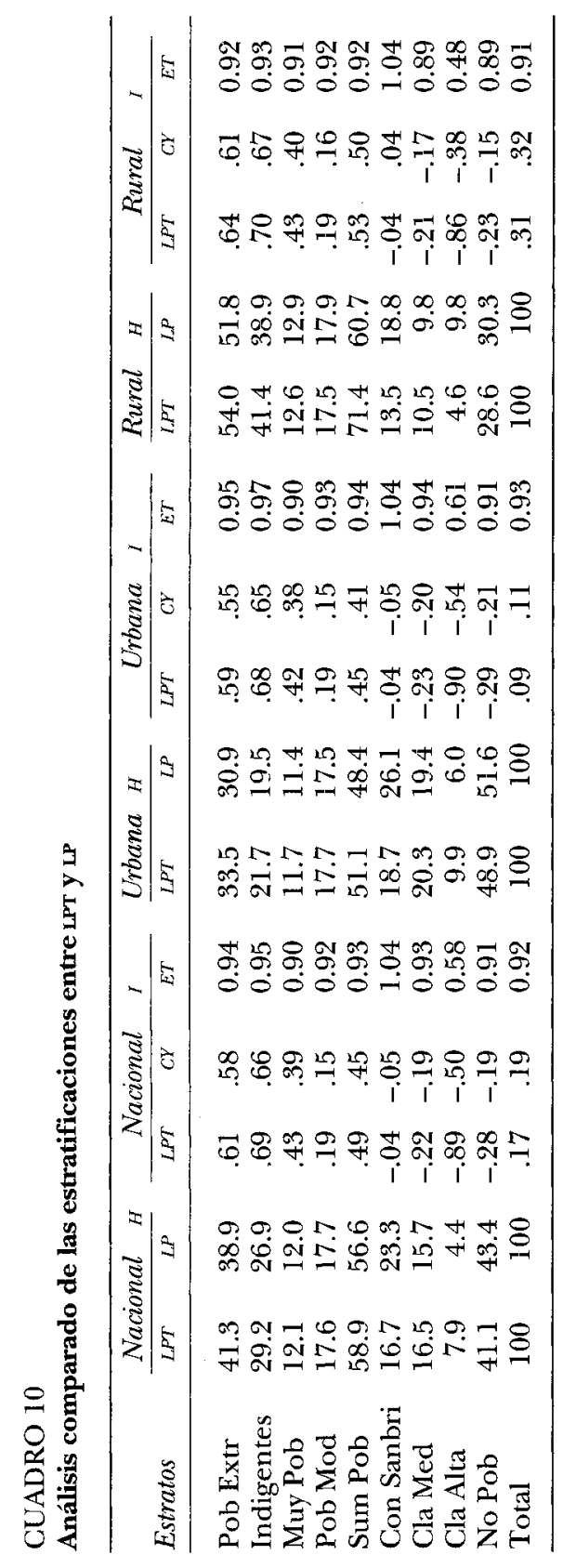


por ingresos corresponde una $\mathrm{I}(\mathrm{LP})$ de 0.45 , a $58.9 \%$ identificado por ingresos-tiempo, corresponde una I(LPT) de 0.49. Es decir, no sólo hay más pobres sino que éstos son más pobres, tanto en el medio urbano como en el rural, aunque en mayor medida en el primero.

Además, el aumento de la intensidad ocurre en todos los estratos de pobres urbanos, rurales y nacionales, independientemente de que la $\boldsymbol{H}$ respectiva haya o no aumentado. Entre los no pobres, que tienen una CY negativa, indicando que tienen ingresos por arriba de la línea de pobreza, y cuyo número se vio disminuido, aumenta el bienestar que los valores negativos de CY y de I(LPT) denotan. Para el conjunto de los no pobres en el ámbito nacional, el cambio es de -0.19 a -0.28 , resultado de incrementos similares entre el medio urbano (de -0.21 a -0.29 ) y el rural (de -0.15 a -0.23). Los cambios más dramáticos, sin embargo, se presentan -al igual que en materia de incidencia- entre la clase alta, que resulta así no sólo más numerosa sino en condiciones de bienestar más elevados. En efecto, para la clase alta en su conjunto, que creció del 4.4 a $7.9 \%$, el nivel de bienestar pasó de -0.50 a -0.89 . El fenómeno mostró un comportamiento similar en el campo que en las ciudades, aunque mucho más marcado en el primero, donde más que se duplicó, al pasar de -0.38 a -0.86 para una clase alta que se vio multiplicada por 2.7, al pasar de 1.7 a $4.6 \%$. Nuestro método LPT, que modifica el método LP, pone al descubierto desigualdades sociales que aquél desconoce y que muestran una sociedad más polarizada.

De todos los indicadores, el que tiene una mayor variabilidad es el de salud y seguridad social, cass. Tanto en el medio urbano como en el rural, va desde más de 0.9 entre los indigentes, hasta -0.95 en la clase alta. Entre los estratos de pobres, el contraste en los valores de cass es tajante: los indigentes por NBI, 0.92 en el medio rural y 0.94 en el urbano; los muy pobres, 0.47 en el rural y 0.66 en el urbano, y los pobres moderados, 0.17 en el rural y 0.25 en el urbano -caso excepcional en que todos los estratos están peor en el medio urbano que en el rural-. Vale la pena destacar la variación muy fuerte entre los pobres: desde 0.9 a alrededor de 0.2 en el indicador.

En otros indicadores, las variaciones entre estratos de pobres son menos marcadas que en cass, pero aun así muy importantes: el rezago educativo de los indigentes rurales $(0.54)$ es más de cinco veces mayor que el de los pobres moderados urbanos $(0.10)$. En hacinamiento los contrastes dentro de los estratos de pobres son menos notables (tres veces): de 0.60 entre los indigentes urbanos a 0.19 entre los pobres moderados rurales. 


\section{Conclusiones}

\section{Conclusiones empiricas y metodológicas}

En primer lugar, debemos concluir que la pobreza en México es un fenómeno mucho más generalizado e intenso que lo que se deriva de otros estudios sobre el tema (INEGI y CEPAL, 1993; CEPAL y PNUD, 1990; Levy, 1991; Lustig y Mitchell, 1994; Banco Mundial, 1993. Para una comparación de algunos de estos resultados, véanse también Hernández Laos, 1992b y Julio Boltvinik, 1996). Aunque rebasa el marco del presente ensayo llevar a cabo un análisis comparativo, como sintesis podemos señalar que: $a$ ) en México no se ha aplicado el método de NBI, de tal manera que todos los autores citados usan solamente el de LP. Lo que los distingue es, por tanto, la forma como definen las líneas de pobreza y de pobreza extrema, el nivel de éstas, y si corrigen o no los datos de ingresos para hacerlos coherentes con cuentas nacionales. La mayoría identifica la pobreza extrema siguiendo la tradición cepalina, con el costo de una canasta alimentaria, la que después multiplica por un factor para obtener la línea de pobreza. Éste es el caso, además de CEPAL y PNUD, de INEGI y CEPAL, y de alguna manera, del Banco Mundial y de Lustig y Mitchell. Todos estos estudios obtienen incidencias de pobreza más bajas, tanto que las de LP-CNSE que hemos presentado en la segunda parte, como las del MMIP presentadas en la tercera parte. En general, las incidencias de la pobreza que obtienen son menores a $50 \%$. Por ejemplo, cEPAL y PNUD obtienen $37.4 \%$ en 1984; el Banco Mundial, 22.6\% en 1989, y el INEGI y CEPAL, $44.1 \%$ en 1992.

En segundo lugar, debemos concluir que, en términos de la evolución de la pobreza, al igual que ocurre con muchos otros aspectos, los últimos treinta años pueden dividirse en dos periodos, teniendo como punto de corte 1981. En efecto, desde 1963 hasta 1981 la pobreza desciende rápidamente en el país, pero cambia su tendencia a partir de 1982: ésta es ascendente y así continúa hasta nuestros días. Tal comportamiento de descenso y ascenso se explica por las políticas económicas diferenciales puestas en vigor en ambos periodos.

En tercer lugar, el análisis de las estructuras de la pobreza en un momento, aplicando el método de medición integrada de la pobreza (ммIP) que, según se argumentó en la primera parte, es el más adecuado para medir el fenómeno, muestra que más de dos terceras partes de la población nacional $(70.6 \%)$ son pobres; que cerca de la mitad de 
ella es pobre extrema (44.7\%); mientras que en el otro polo, $11.4 \%$ de la población pertenece a la clase media y sólo $5.5 \%$ a la clase alta.

En cuarto lugar encontramos que, en general, la pobreza disminuye con el tamaño de la localidad, salvo al pasar a la Zona Metropolitana de la Ciudad de México (zмcm) donde se encontró una inciden cia de la pobreza más alta que en las otras ciudades de más de 500000 habitantes. Más de la mitad de la población pobre del país habita en zonas de alta densidad (urbanas), hallazgo que contrasta con el de otros estudios como el del Banco Mundial (1993) que consideran que el problema de la pobreza en México es fundamentalmente rural. Esta conclusión se ve matizada, sin embargo, cuando encontramos que la intensidad media de la pobreza es sustancialmente más alta en el medio rural que en el urbano, lo cual permite afirmar que si bien hay más pobres en el ámbito urbano que en el rural, los de este último son más pobres aún.

En términos de las participaciones de ambos medios en el indicador compuesto HI, que expresa la masa carencial, y cuyo análisis no fue incluido en el texto por falta de espacio (véase, sin embargo, el cuadro 6), el área de baja densidad representa la mayor parte de la masa carencial del país. Esto permite concluir que si bien hay más per. sonas pobres en el área urbana, hay más pobreza en la rural.

En quinto lugar, la pobreza es heterogénea en varios sentidos. Por una parte, por la intensidad variable de la pobreza que en este estudio nos ha permitido distinguir tres estratos de pobreza, que también hemos agrupado en dos. Por otra parte, porque encontramos varios grupos de pobres según el tipo de carencias que padecen: carencia de ingresos-tiempo por una parte, y de necesidades básicas por la otra. Esta última se puede expresar de manera agregada como pobreza por NBI, y de manera desagregada como carencias específicas, habiendo observado que la carencia más intensa es la de salud y seguridad social. $\mathrm{Al}$ combinar ambas dimensiones, tipo de pobreza e intensidad, obtuvimos las categorías de pobres totales, pobres extremos totales, e indigentes totales, para aquellos hogares que padecen carencias en las dos dimensiones básicas, y pudimos cuantificar su importancia. Igualmente distinguimos los pobres sólo por NBI y sólo por LPT. La pobreza de cada uno de estos grupos tiene orígenes distintos y requiere soluciones diversas, por lo que estrictamente no bastaría una política de lucha contra la pobreza, sino varias. La capacidad para mostrar esta heterogeneidad y, por tanto, derivar políticas diversificadas es una de las virtudes exclusivas del MMIP. 
En sex to lugar, desde la perspectiva del análisis metodológico comparado, pudimos contrastar el nivel de incidencia e intensidad de la pobreza que se obtiene con el MMrP contra otros tres resultados: la versión inicial de éste a la que denominamos MMIP matricial, y los dos métodos parciales (NBI y LPT) con los que se construye el MMIP. Constatamos que, a diferencia del MMIP matricial (o LP-NBI), que necesariamente resulta en grados de pobreza más altos que los métodos parciales, el MMIP tiende más bien a ubicarse en una posición intermedia entre ambos procedimientos parciales como consecuencia de lo que había previsto (J. Boltvinik, 1992: 358-359) respecto de la compensación de ciertas carencias, dentro de un hogar, con situaciones por arriba de la norma en otras dimensiones. Algo similar ocurre respecto de la intensidad de la pobreza.

\section{Bibliografía}

Alarcón, Diana (1994), Changes in the Distribution of Income in Mexico and Trade Liberalization, Tijuana, El Colegio de la Frontera Norte.

Banco Mundial (1993), Poverty and Income Distribution in Latin America. The Story of the Eighties, Washington.

Boltvinik, Jana, Jorge Creixell, Mari Carmen Díaz, Regina García Cuéllar y Antonio López Puerta (1994), "Estimación de la pobreza y la pobreza extrema en México. Ajuste a cuentas nacionales 1992", México, IтAм (mimeo).

Boltvinik, Julio (1984), "Satisfacción desigual de las necesidades esenciales en México", en Carlos Tello y Rolando Cordera (eds.), La desigualdad en México, México, Siglo XXI Editores, pp. 17-64.

_ (1986), "Sistema de necesidades y modo de vida en México", Investigación Económica, vol. 45, núm. 175, Facultad de Economía, unam, pp. 169-204.

(1986a), "Modo de producción estatal y satisfacción de necesidades esenciales en México", Investigación Económica, vol. 45, núm. 177, pp. 195-244.

(1990), Pobreza y necesidades bâsicas. Conceptos y métodos de medición, $\mathrm{Ca}-$ racas, Programa de las Naciones Unidas para el Desarrollo (PNUD).

- (1992), "El método de medición integrada de la pobreza. Una propuesta para su desarrollo", Comercio Exterior, vol. 42, núm. 4, pp. 354-365.

(1994), "La satisfacción de las necesidades esenciales en México en los setentas y ochentas", en Pablo Pascual y José Woldenberg (eds.), Desarrollo, desigualdad y medio ambiente, México, Cal y Arena, pp. 99-175.

(1995), Pobreza y estratificación social en México, Aguascalientes, INEGI.

(1996), "Diversas visiones sobre la pobreza en México", Diálogos, Caracas, núms. 18-19, pp. 16-17. 
(1997), "Incidencia e intensidad de la pobreza en México. 1989", en J. Boltvinik, J. y E. Hernández Laos (eds.), La pobreza y distribución del ingreso en México, México, El Colegio de México (en prensa).

CEPAL Y PNUD (1990), Magnitud de la pobreza en América Latina en los años ochenta, Santiago de Chile.

Coplamar (1982), Necesidades esenciales y estructura productiva en México, anexo, México, Presidencia de la República.

(1983), Macroeconomía de las necesidades esenciales en México, México, Siglo XXI Editores.

Gobierno de la República de Bolivia (1993), Mapa de pobreza. Una guía para la acción social, anexo metodológico, La Paz, Ministerio de Desarrollo Humano.

Hernández Laos, Enrique (1992), Crecimiento económico y pobreza en México, México, UNAM.

- (1992b), "La pobreza en México", Comercio Exterior, vol. 42, núm. 4, pp. 402-411.

INEGI (1992), Encuesta nacional de ingresos y gastos de los hogares, 1989, México.

INEGI y CEPAL (1993), Magnitud y evolución de la pobreza en México. 1984-1992. Informe metodológico, Aguascalientes.

Kaztman, Rubén (1989), "La heterogeneidad de la pobreza. El caso de Montevideo", Revista de la CEPAL, núm. 37, Santiago de Chile.

Levy, Santiago (1991), Poverty Alleviation in Mexico, Washington, Banco Mundial. Lustig, Nora y Ann Mitchell (1994), "Poverty in Times of Austerity: Mexico in the 1980's”, trabajo presentado en la XII Reunión Latinoamericana de la Econometric Society, 2-5 de agosto, Caracas.

\section{Lista de abreviaturas utilizadas en el texto y en los cuadros}

CAss: carencia de atención en salud y seguridad social CBD: carencia en bienes durables o en patrimonio básico CCEv: carencia en la calidad y espacios de la vivienda CCV: carencia en la calidad de la vivienda (materiales de construcción y recubrimientos)

CNSE: canasta normativa de satisfactores esenciales cNss: canasta normativa de satisfactores de subsistencia

Coplamar: Coordinación General del Plan Nacional de Zonas Deprimidas y Grupos Marginados

Cots: carencia en otros servicios (electricidad y teléfono)

cs: carencia sanitaria (agua, drenaje y baño)

cx: Carencia de ingresos

ENIGr: Encuesta Nacional de Ingresos y Gastos de los Hogares

ET: exceso de trabajo

r: incidencia de la pobreza (proporción de personas pobres en la población) 
$\mathrm{H}($ IndigTot): incidencia de la indigencia total (indigencia simultánea en ambas dimensiones)

н(LP): incidencia de la pobreza por el método de LP H(LPT): incidencia de la pobreza por el método de LPT н(мміP): incidencia de la pobreza por el мMіP $\mathrm{H}(\mathrm{NBI})$ : incidencia de la pobreza por el método de NBI H(PobExtTot): incidencia de la pobreza extrema total (pobreza extrema simultánea en ambas dimensiones

$\mathrm{H}($ PobTot): incidencia de la pobreza total (hogares y personas en pobreza simultánea en ambas dimensiones: NBI y LPT)

HI: grado de pobreza, producto de la incidencia por la intensidad de la pobreza HMD: hacinamiento multidimensional (que toma en cuenta cocina, dormitorios y cuartos multiusos)

1(LPT): intensidad de la pobreza en la dimensión (o método) de LPT I(MMIP): intensidad de la pobreza por el MMIP 1(NBI): intensidad de la pobreza en la dimensión (o método) de NBI INPC: índice nacional de precios al consumidor LP: línea de pobreza

LPT: [método de] línea de pobreza-tiempo

LPT-NBI: sinónimo del MMIP matricial

Metrop: ciudades de 500000 y más habitantes MMIP: método de medición integrada de la pobreza

MMIP matricial: versión del método de medición integrada de la pobreza, según la cual son pobres los hogares así considerados por LP O por NBI o por ambos

NBI: [método de] necesidades básicas insatisfechas

NBS: necesidades básicas satisfechas

No metrop: ciudades menores a 500000 habitantes

RE: rezago educativo (combinación de grados educativos y alfabetismo)

Sanbri: estrato que cuenta con satisfacción de necesidades básicas y de requerimientos de ingresos

SRI: satisfacción de requerimientos de ingresos

zмсм о амсм: Zona o Área Metropolitana de la Ciudad de México 